\title{
A FUNCTIONAL ANALYSIS MODEL FOR NATURAL IMAGES PERMITTING STRUCTURED COMPRESSION
}

\author{
JACQUES Froment ${ }^{1,2}$
}

\begin{abstract}
This paper describes a compact perceptual image model intended for morphological representation of the visual information contained in natural images. We explain why the total variation can be a criterion to split the information between the two main visual structures, which are the sketch and the microtextures. We deduce a morphological decomposition scheme, based on a segmentation where the borders of the regions correspond to the location of the topological singularities of a topographic map. This leads to propose a new and morphological definition of edges. The sketch is computed by approximating the image with a piecewise smooth non-oscillating function, using a Lipshitz interpolant given as the solution of a PDE. The data needed to reconstruct the sketch image are very compact, so that an immediate outcome of this image model is the design of a progressive, and artifact-free, image compression scheme.
\end{abstract}

Résumé. Cet article décrit un modèle perceptuel et compact, destiné à donner une représentation morphologique de l'information visuelle contenue dans les images naturelles. Nous expliquons pourquoi la variation totale peut être un critère pour séparer l'information entre les deux structures essentielles, qui sont le sketch et les micro-textures. Nous en déduisons un procédé de décomposition morphologique, basé sur une segmentation où les bords des régions correspondent à la position des singularités topologiques de la carte topographique. Cela nous permet de proposer une définition des bords, nouvelle et morphologique. Le sketch est calculé en approchant l'image par une fonction nonoscillante et régulière par morceaux, en utilisant une interpolation Lipschitz donnée comme la solution d'une EDP. Les données nécessaires à la reconstruction de l'image sketch étant très compactes, une application immédiate de ce modèle est la mise au point d'un procédé de compression progressive des images sans déformation visuelle.

AMS Subject Classification. 68U10, 26B30, 35Q80.

Received November 23, 1998. Revised May 31, 1999.

\section{INTRODUCTION}

One of the main issue for image analysis is to define a mathematical representation of the image that offers suitable properties to build most of the computer vision algorithms. By using the same words than Marr [24], the problem is to determine the primitives or atoms of the image, in order to constitute a raw primal sketch.

What properties should have a perfect raw primal sketch?

\footnotetext{
Keywords and phrases: Perceptual image model, total variation, mathematical morphology, segmentation, image compression.

${ }^{1}$ PRISME, UFR de Math. et Info., Université R. Descartes Paris 5, 45 rue des Saints-Pères, 75270 Paris Cedex 06, France.

${ }^{2}$ CMLA, École Normale Supérieure de Cachan, 61 avenue du Président Wilson, 94235 Cachan Cedex, France;

e-mail: Jacques.Froment@math-info.univ-paris5.fr Jacques.Froment@cmla.ens-cachan.fr
}

(c) EDP Sciences, SMAI 1999 
1. Structure: the atoms must capture and match the structures in the images, which basically can be separated into edges surrounding homogeneous regions and microtextures which characterize the regions.

2. Progressive representation: the set of all atoms must allow the reconstruction of an image visually identical to the original. By using the most representative atoms only, one has to be able to reconstruct an image closed to the original, and the missing atoms should not produce visual artifacts on the reconstructed image.

3. Invariances: the primal sketch should not depend on the particular condition of the way the image has been captured by a camera. This implies invariance under translation, rotation, symmetry (arbitrary position of the camera) and zoom (change of scale), i.e. invariance by affine operators. Another important property is the invariance under change of contrast, also called morphological invariance: the light captors of the cameras are nonlinear nondecreasing functions, and these functions differ from one camera to the other (the response of a given camera is even changing with time). In the Gestalt theory [35], the invariance under change of contrast is one of the reconstruction principles used to elaborate a comprehensive representation. In the following, we shall use the term morphology for the contrast invariance property.

4. Compactness: to obtain efficient algorithms and to allow image compression, the primal sketch must lead to a compact data set.

5. Computable property: a fast algorithm to compute the atoms is required.

The raw primal sketch of Marr is based on the detection of the intensity changes in the image, by recording the zero-crossing location of the image filtered by the Laplacian of the Gaussian at a given scale. The edges are then defined as discontinuity lines, and the scale parameter allows to discriminate the important atoms. This approach has been successfully developed in the past, since it meets almost all the requirements of a "good primal sketch" (see e.g. [18] for a reconstruction algorithm and [4] for an optimal edge detector). These last years have seen interesting reformalizations of this multiscale edges representation, in a wavelet [23] and in a variational [31] framework.

However, the multiscale edges representation still suffers for some drawbacks that do not make it always suitable for image analysis: the representation is not invariant under change of contrast. This means that the edges locations of an image on which a change of contrast has been applied differ from the original edges locations. In addition, the Fourier or wavelet transform applied to compute the atoms leads to visual artifacts on the reconstructed image, due to the quantization and sampling of the coefficients needed to get a compact representation.

More recently, it has been proved in [2] that, under fairly conditions (including all invariances), there exists only one regular multiscale analysis, the so-called AMSS (for Affine Morphological Scale Space). An image is decomposed into this scale-space using a parabolic evolution equation, for which viscosity solutions [13] exist. Because of the morphological invariance, the evolution of the image along the scales is equivalent to the evolution of its level curves, which are defined as the border of the level sets. A level set is a set of pixels with gray levels below (or above) a given threshold.

The representation of an image by its level sets has been proposed by the Mathematical Morphology school $[26,33]$ as a geometrical decomposition which offers the contrast invariance. This representation can be viewed as another raw primal sketch, for which all the properties stated before are met but the compactness. Recently, such a decomposition based on the connected components of the level lines has been described [29], together with a fast algorithm. This representation is well adapted to number of image analysis problems (as pattern matching) but it still suffers of a relatively large amount of data. Using AMSS, a simplification of the image can be performed to reduce the amount of data. However, the structure of the image is then considerably weakened and the filtered image does not sound natural.

In this paper, we introduce another model based on the level sets that can be coded using a very small amount of data. This model is also supposed to split the information between edges and microtextures. We show in Section 2 how the total variation can be related to the notion of microtextures: microtextures correspond to fast oscillating parts, and therefore are associated to high variations. On the other hand, edges surrounding flat 
regions generate little variations. The total variation can be expressed in terms of level sets, leading us to link this morphological approach with the edge and microtexture structures.

In order to catch the important features of the images, we select the most perceptive level sets according to the principle affirmed in $[7,9]$, namely that "only pieces of level lines of the image joining junctions are the atoms of visual perception". The importance of the level line junctions for the visual perception is explained in Section 3 , together with the algorithm defined in $[7,9]$ to detect them on digital images.

Only a small subset of all level sets are kept in this way. To improve the quality of the representation, image values in the border of these level sets are also recorded. This composes our image model suitable to represent the lower part of the total variation, which consists of edges surrounding flat regions. This model is described in Section 4.

From these data, it is possible to reconstruct an image close to the original but without the microtexture information. We use the morphological interpolation algorithm given in [8], which is based on the Absolutely Minimizing Lipschitz Extension (AMLE) model. This model ensures a Lipschitz reconstruction between the remaining level lines. Its principle is recalled in Section 5.

A straightforward application of this compact image representation is the design of a compression scheme that respects the human visual system. In addition to the geometrical sketch coding, we propose a compression scheme adapted to the microtexture information. We first use a refinement algorithm to remove any undesirable structure in the texture image, and we compress the remaining information using a wavelet scheme based on the EZW method [34]. This process is described in Section 6. In Section 7, several examples of such compact representations are given.

\section{TOtAL VARIATION AND THE STRUCTURE OF NATURAL IMAGES}

The total variation is a well-known mathematical tool for image processing, but it has been until now essentially used for denoising purposes. The idea, first developed by Osher and Rudin in [32], is to restore a noisy image by smoothing it while preserving its edges, which correspond to discontinuities. This can be done by decreasing the total variation of the image, in such a way that important discontinuities may be reduced but should not disappear [14].

Let $\Omega$ be an open bounded subset of $\mathbb{R}^{2}$. The total variation of an image $u: \Omega \rightarrow \mathbb{R}$ can be simply defined, if $u \in C^{1}(\Omega)$, as

$$
\operatorname{TV}(u)=\int_{\Omega}|\nabla u(x)| d x
$$

with

$$
|\nabla u|=\left|\frac{\partial u}{\partial x_{1}}\right|+\left|\frac{\partial u}{\partial x_{2}}\right|
$$

If the gradient of $u, \nabla u$, does not exist or is not continuous but if $u \in L^{1}(\Omega),(1)$ is generalized into

$$
\operatorname{TV}(u)=\sup _{\phi}\left\{\int_{\Omega} u(x)(\operatorname{div} \phi)(x) d x / \phi \in C_{c}^{1}\left(\Omega, \mathbb{R}^{2}\right) \text { and }|\phi| \leq 1\right\}
$$

where

$$
\operatorname{div} \phi=\frac{\partial \phi_{1}}{\partial x_{1}}+\frac{\partial \phi_{2}}{\partial x_{2}} .
$$

We say that $u$ is of bounded variation $(u \in \operatorname{BV}(\Omega))$ if $\operatorname{TV}(u)<+\infty$. 
Let us denote $L_{\lambda}$ the lower level set $\lambda$ of $u$

$$
L_{\lambda}=\{x \in \Omega / u(x)<\lambda\}
$$

$\partial L_{\lambda}$ the topological boundary of $L_{\lambda}$, and $\partial^{*} L_{\lambda}$ the reduced boundary of $L_{\lambda}$ [15]. If $u$ is BV, then $\partial^{*} L_{\lambda}$ is an union of Jordan curves, those curves being closed or touching the image border $\partial \Omega$. They are the "level lines" of $u$. The perimeter of $L_{\lambda}$, defined as $\operatorname{per}\left(L_{\lambda}\right)=\mathrm{TV}\left(\mathbb{I}_{L_{\lambda}}\right)$, is finite and the coarea formula [15] allows to link the TV of $u$ with the total length of its level lines:

$$
T V(u)=\int_{\mathbb{R}} \operatorname{per}\left(L_{\lambda}\right) d \lambda
$$

Conversely, if $u \in L^{1}(\Omega)$ and $\int_{\mathbb{R}} \operatorname{per}\left(L_{\lambda}\right) d \lambda<+\infty$, then $u$ is BV.

\subsection{Image decomposition between texture and sketch}

Since any physical image is a bounded function, it follows from (1) and (3) that an image $u$ is not BV if it oscillates rapidly or, from the coarea formula, if it has too many small level lines. For example, $(x, y)$ $\rightarrow \sin (1 / x) \notin \mathrm{BV}(] 0,1\left[^{2}\right)$ but $(x, y) \rightarrow \sin (x) \in \mathrm{BV}(] 0,1\left[^{2}\right)$.

What structure should perceive somebody seeing such non BV images? The fast oscillations create repetition of small patterns at finer and finer scales: the observer has the illusion of microtextures. The terms of texture and microtexture are not well defined, mainly because one has to link a perceptual feeling to an abstract object. However, one considers usually that the notion of texture is related to a characteristic repetition in a given area, microtexture corresponding to high frequencies repetition of small primitives.

In the continuous or analog model, the image resolution is not finite and one may observe more details by moving closer. If the repetition disappears at some scale, the observer has no longer the illusion of a microtexture: he perceives the primitives as distinct objects.

The perception of microtextures is therefore somewhat equivalent to the existence of fast oscillations at every scale. Conversely, structures which are not perceived as microtextures may be interpreted as objects, composed by edges surrounding homogeneous regions.

These remarks lead us to give the following definition:

Definition 1. We call BV-decomposition of an image $u \in L^{1}(\Omega), u \notin B V(\Omega)$, any pair of functions (v,w) defined on $\Omega$ such that $v, w \in L^{1}(\Omega), v \in B V(\Omega)$ and

$$
u=v+w \quad \text { almost everywhere in } \Omega \text {. }
$$

The function $v$ is called sketch of the image $u$ while $w$ is the microtexture.

This definition is not enough to get a notion of sketch and microtexture consistent with the visual system and with the original image $u$ : if $(v, w)$ is a BV-decomposition, $(v+s, w-s)$ with $s \in \mathrm{BV}(\Omega)$ is another BV-decomposition of $u$, although $v$ and $v+s$ (as well as $w$ and $w-s$ ) may be very different. We shall see in the next section how a decomposition based on a segmentation can be made consistent.

In our former observations, we did not consider the case of an image having too many level sets of infinite perimeter and which is, therefore, not BV. This may happen when the borders are very irregular, for example when they have a fractal structure. More frequently on natural images, level sets with irregular borders may arise in low contrasted regions (as the gradation in light intensity on an uniformly shined surface) because of limited accuracy in the physical device used to capture the image. Such level sets do not reveal a microtexture nor a sketch information, they are rather non-perceptive structures that may be neglected. We will see in Section 6 how to remove this irrelevant information from the sketch and the microtexture images of a BV-decomposition. 

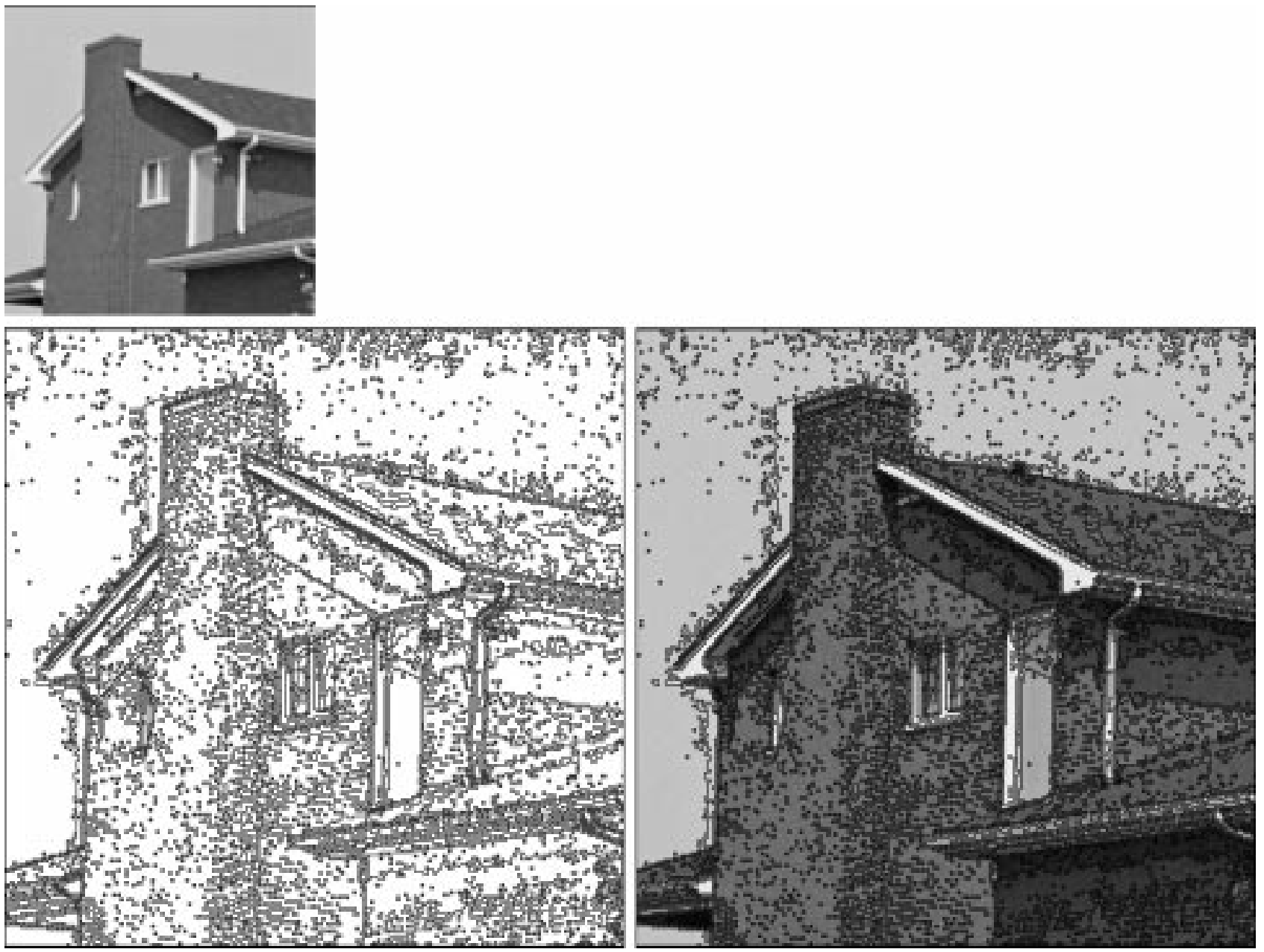

Figure 1. A topograpic map. Up: original "House" image $(256 \times 256$ pixels, 256 gray levels $)$. Down and left: level lines location for levels $\lambda_{1}=20, \lambda_{2}=40, \lambda_{2}=60, \ldots, \lambda_{12}=240, \lambda_{13}=$ $+\infty$. The image size is doubled since, in the discrete case, the level lines lie between the pixels grid. Down and right: same topographic map with the zoomed original image in background.

\subsection{Morphological decomposition based on a segmentation}

If a function is not of bounded variation in a given subset $\Omega^{\prime} \subset \Omega$, it will not be of bounded variation on any subset containing $\Omega^{\prime}$. Therefore, the natural way to get a morphological representation is to segment $\Omega$ into subsets $\Omega^{\prime}$ where $u \in \mathrm{BV}\left(\Omega^{\prime}\right)$ and subsets $\Omega^{\prime \prime}$ where $u \notin \mathrm{BV}\left(\Omega^{\prime \prime}\right)$, using subsets based on level sets. If we map the level lines of an image for a given set of levels $\left\{\lambda_{1}<\lambda_{2} \ldots<\lambda_{n}=+\infty\right\}$, we get a segmentation of the image with sets of type $\left\{x \in \Omega / \lambda_{i-1}<u(x)<\lambda_{i}\right\}$, also called topographic map [9] (see Fig. 1).

More generally, one can consider a segmentation achieved using only some connected components of lower levet sets $\left(L_{\lambda}\right)_{\lambda}$ and upper level sets $\left(M_{\mu}\right)_{\mu}$, where the upper level set $\mu$ of $u$ is

$$
M_{\mu}=\{x \in \Omega / u(x)>\mu\}
$$

We shall call level set any lower or upper level set. 
Our intention is to approximate the image by BV functions smooth all the way up to the boundary of some level sets. This necessitates some assumptions on the geometric behavior of these boundaries. We say that a set $P \subset \Omega$ has a Lipschitz border if $\partial P$ is locally the graph of a Lipschitz function [15]. In that case, $\operatorname{per}(P)=\mathcal{H}^{1}(\partial P)$ : the perimeter of $P$ can be interpreted as the length of the boundary of $P$.

Definition 2. Let $\mathcal{S}$ be a family of connected components of level sets with $\Omega \in \mathcal{S}$. We call morphological segmentation of $u$ the set $\mathcal{P}=\left(\mathcal{P}_{i}\right)_{i=1, \ldots, n}$ defined by the topographic map of $\mathcal{S}$ (each $P_{i}$ being the interior of a region of this map) if this set is finite with each region of Lipschitz border. In that case, we note $\mathcal{P}=M S(\mathcal{S})$.

We easily get the following properties:

\section{Proposition 1.}

$$
\begin{aligned}
& -\quad P_{i} \neq \emptyset, \\
& -\quad \text { interior }\left(\bigcup_{i=1}^{n} \overline{P_{i}}\right)=\Omega, \\
& -\quad i \neq j \Longrightarrow P_{i} \cap P_{j}=\emptyset, \\
& -\quad \partial \bigcup_{i=1}^{n} P_{i} \text { is Lipschitz (but for a finite number of points). }
\end{aligned}
$$

A morphological segmentation is shown in Figure 2. In our framework, the pertinence of a morphological segmentation is related to the choice of the sequences $\left(\lambda_{i}\right)_{i}$ and $\left(\mu_{i}\right)_{i}$ so that the sets $P_{i}$ match as well as possible the visual perception of the important structures. This issue will be addressed in the Section 3 .

The following result gives an example of an image decomposition based on a morphological segmentation:

Proposition 2. Let $\mathcal{P}=\left(\mathcal{P}_{i}\right)_{i=1, \ldots, n}$ be a morphological segmentation of $u$. We define

$$
\left\{\begin{array}{l}
v=r_{i} \text { on } P_{i} \text { where } r_{i} \in B V\left(P_{i}\right) \\
w=u-v
\end{array}\right.
$$

Then, $(v, w)$ is a $B V$-decomposition of $u$.

Proof. Let us prove that the function $v$ defined on $\Omega$ by $v_{\mid P_{i}}=r_{i}$ belongs to $B V(\Omega)$ : take $\phi \in C_{c}^{1}\left(\Omega, \mathbb{R}^{2}\right)$, $|\phi| \leq 1$. Since $P_{j} \cap P_{k}=\emptyset$, we have

$$
\int_{\Omega} v \operatorname{div} \phi d x=\sum_{i=1}^{n} \int_{P_{i}} r_{i} d i v \phi d x=-\sum_{i=1}^{n} \int_{P_{i}} \phi \sigma_{i} d\left\|D r_{i}\right\|+\sum_{i=1}^{n} \int_{\partial P_{i}}\left(\phi \nu_{P_{i}}\right) T_{r_{i}} d \mathcal{H}^{1}
$$

where

- $\nu_{P_{i}}$ is the outer unit normal of $\partial P_{i}$, which exists $\mathcal{H}^{1}$-a.e. on $\partial P_{i}$ according to Rademacher's theorem [15],

- $\left\|D r_{i}\right\|$ is the variation measure of $r_{i}$ on $P_{i}$,

- $\sigma_{i}: P_{i} \rightarrow \mathbb{R}^{2}$ is a measurable function defined by the Riesz representation theorem [15], such that $\left|\sigma_{i}(x)\right|=$ $1\left\|D r_{i}\right\|$-a.e.,

- $T_{r_{i}}$ is the trace of $r_{i}$ on $\partial P_{i}$. Notice that $T: r_{i} \in B V\left(P_{i}\right) \rightarrow T_{r_{i}} \in L^{1}\left(\partial P_{i}, \mathcal{H}^{1}\right)$ is a bounded linear mapping [15].

Therefore,

$$
\left|\int_{\Omega} v \operatorname{div} \phi d x\right| \leq \sum_{i=1}^{n} \int_{P_{i}} d\left\|D r_{i}\right\|+\sum_{i=1}^{n} \int_{\partial P_{i}}\left|T_{r_{i}}\right| d \mathcal{H}^{1} \leq \sum_{i=1}^{n}\left\|D r_{i}\right\|\left(P_{i}\right)+\sum_{i=1} c_{i}\left\|r_{i}\right\|_{B V\left(P_{i}\right)}=C<+\infty .
$$

And thus, $T V(v)=\|D v\|(\Omega)<+\infty$.

At last, since $v \in B V(\Omega), u \notin B V(\Omega)$ and $u=v+w$, it follows that $w \notin B V(\Omega)$.

As an example, we may simply chose $r_{i}=u_{\mid P_{i}}$ if $u_{\mid P_{i}} \in \mathrm{BV}\left(P_{i}\right)$ and 0 elsewhere. However, from a perceptual point of view, it is better to take a smooth $\mathrm{BV}$ function that approximates $u$ on the regions where $u$ contains 

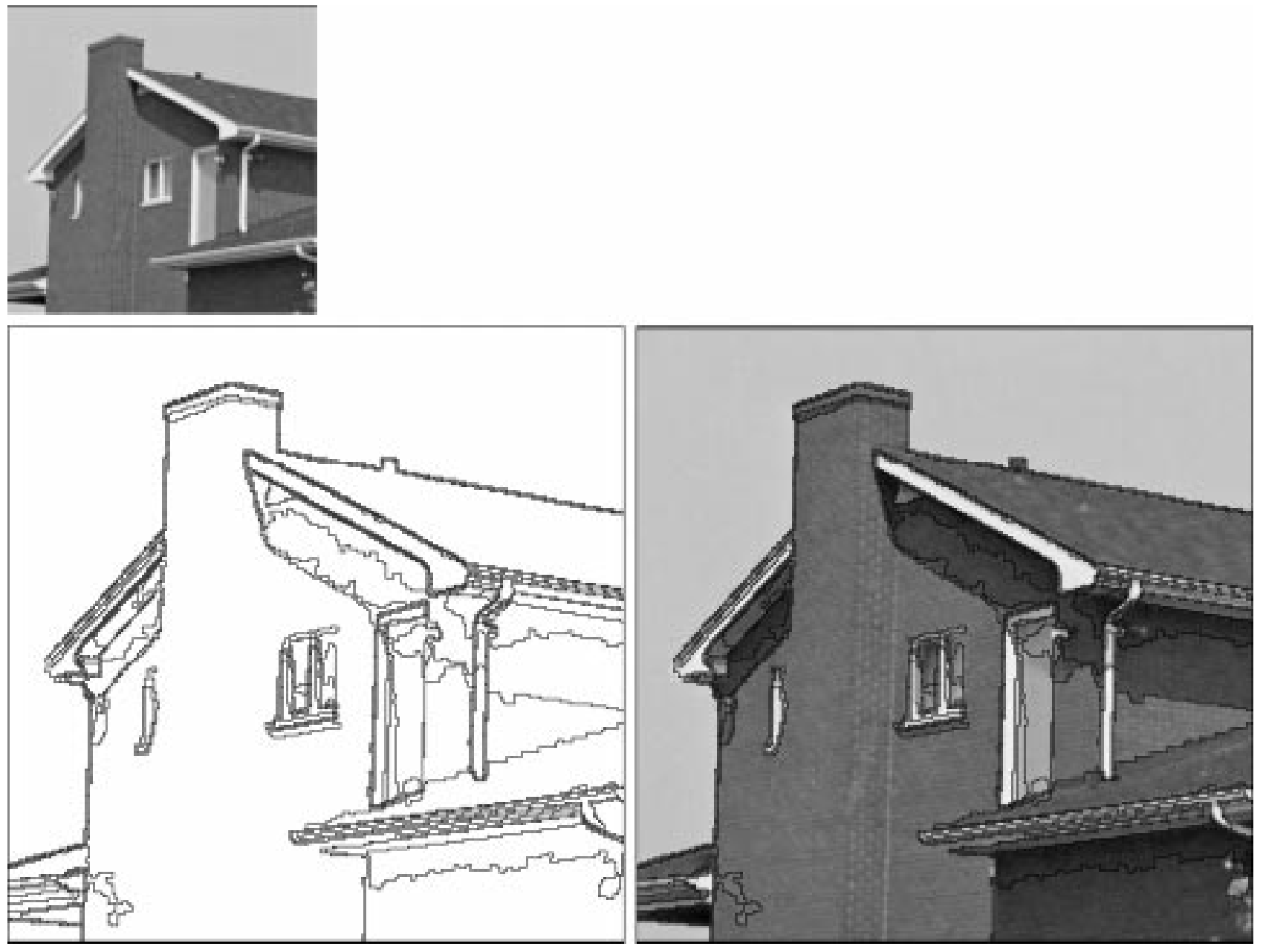

FIGURE 2. A morphological segmentation obtained using the algorithm described in Section 4.1, in order to get cells matching the important structures. Up: original "House" image. Down and left: the morphological segmentation. Down and right: the morphological segmentation together with the zoomed original image.

the microtextures. In this way, one can separate the local tendency of the image, which is part of the sketch, from the high frequencies component which generates the microtexture. Moreover, the choice $r_{i} \neq u_{\mid P_{i}}$ allows to approximate the BV regions of $u$ by smooth functions. Remember that in these places, the image is mainly composed by edges surrounding homogeneous regions: if the morphological segmentation is computed so that the borders $\partial P_{i}$ correspond to the location of the most important discontinuities of $u$, it is possible to approximate $u$ inside $P_{i}$ by a smooth function with good accuracy.

Definition 3. A BV-decomposition $(v, w)$ of $u$ is called morphological decomposition if it is obtained by Proposition 2 with a choice of a segmentation $\mathcal{P}=\left(\mathcal{P}_{i}\right)_{i=1, \ldots, n}$ and of functions $\left(r_{j}\right)_{j \in J}$ and $s$ that ensures a good approximation of $u$ by $v$ (e.g. a relatively small $\|w\|_{1}$ ). 


\subsection{The discrete case}

Since our study must result in practical algorithms, we shall now switch from the continuous to the discrete case where $u: \Omega \subset \mathbb{Z}^{2} \rightarrow \mathbb{Z}$. The definition of a morphological decomposition must be adapted to the discrete case: digital images are of finite resolution, and therefore all digital images are BV. It follows from above that, if $v$ is a piecewise smooth and non-oscillating approximation of $u$ on each region defined by the topographic map, $(v, u-v)$ is a morphological decomposition of $u$. We shall extend this result in the case where $u \in \operatorname{BV}(\Omega)$ by proposing the following algorithm:

\section{Discrete morphological decomposition}

Step 1: Compute a morphological segmentation $\mathcal{P}=\left(\mathcal{P}_{i}\right)_{i=1, \ldots, n}$ of the digital image such that $\left(\partial P_{i}\right)_{i=1, \ldots, n}$ matches the borders of the most important structure;

Step 2: on each region $P_{i}$, compute a smooth and non-oscillating approximation of the image. Let $v$ be the resulting sketch image;

Step 3: compute the microtexture image $w=u-v$.

In the next sections, we shall see how to solve the first step of this algorithm. We will use the "atoms of the perception", which are junctions between level lines, to choose the most representative regions. Since we are seeking at the end a compact image representation, the approximation $v$ has to be computed using a small amount of data. The data will be samples of the image values at the borders $\partial P_{i}$. Section 4 will explain in more details this compact image representation, while in Section 5 we shall recall the AMLE model, which is used to compute the second step of the algorithm.

Notice that in the discrete case $\Omega \subset \mathbb{Z}^{2}$, the border of a region lies in the shifted grid $(\mathbb{Z}+1 / 2)^{2}$. To overcome this problem, we shall introduce the following notations, where $B(x, r)$ is the closed disk of center $x$ and radius $r$ :

Definition 4. We note $\partial^{I} P$ the internal border of a region $P$ that is, the set

$$
\partial^{I} P=\{x \in P / \exists y \notin P / y \in B(x, \sqrt{2})\}
$$

Similarly, $\partial^{E} P$ is the external border of $P$ :

$$
\partial^{E} P=\{x \notin P / B(x, \sqrt{2}) \cap P \neq \emptyset\} .
$$

Figure 3 displays a region in the discrete grid together with its internal and external borders. Notice that the geometrical information of $\partial P$ is equivalent to the pair $\left(\partial^{I} P, \partial^{E} P\right)$ that is, there exists obvious algorithms to switch from one representation to the other one. Since $P$ is a region of a morphological segmentation, we get the following properties:

\section{Proposition 3.}

$$
\begin{aligned}
& \text { - } \forall P_{i} \in \mathcal{P}, \forall x \in \partial^{I} P_{i}\left(x \notin \partial^{I} \Omega\right), \exists P_{j} \in \mathcal{P} / x \in \partial^{E} P_{j} \\
& \text { - } \forall P_{i} \in \mathcal{P}, \forall x \in \partial^{E} P_{i}, \exists P_{j} \in \mathcal{P} / x \in \partial^{I} P_{j}
\end{aligned}
$$

\section{The ATOMS OF THE PERCEPtion}

How should be computed a morphological segmentation $\mathcal{P}$ such that the borders of the most important structures are located in the regions $P_{i}$ ? A classical answer would be to try to match the edges, in the sense of the discontinuity lines in the image. However, the use of classical edge detectors is not consistent with the morphological approach, as it is well explained in [7,9], essentially because such edges are not contrast invariant. In this paper, Caselles, Coll and Morel argue that the atoms of the perception, that is, the basic elements on which further representations may be built, are not edges but "pieces of level lines joining junctions". Indeed, 


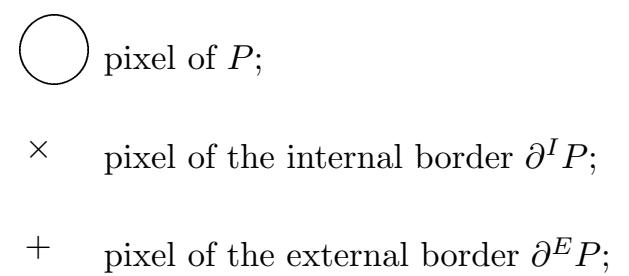

— border $\partial P$ (does not belong to the pixels grid).

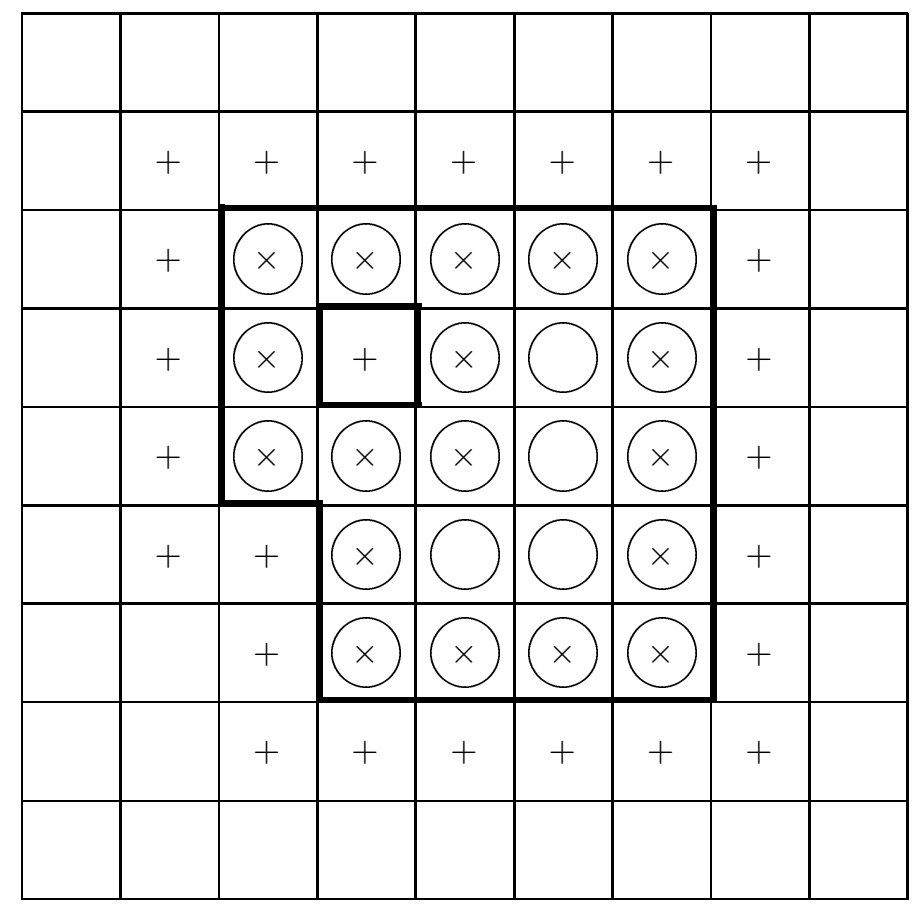

Figure 3. Example of a region $P$ in the discrete grid.

the physical generation process of an image implies some events (as occlusions and transparencies) which cause singularities on the topographic map: level lines joining some other level lines with a shape (more or less) like a $\mathrm{T}$ in case of an occlusion. Such T-junctions are the most important junctions and other types of junctions will not be considered here.

The T-junction singularity is one of the most significant principles of the visual reconstruction, which allow a geometrical constitution of the visual objects. It is in the heart of the Gestaltists' theory, and in particular of the Kanizsa's work $[19,20]$. Each time a T-junction is detected, our perception reconstructs the occlusion of an object by another one, and the border of the occluded object is mentally extended behind the horizontal bar of the T. In the second drawing of Figure 4, the observer reconstructs black disks from quarters of disks only. This phenomenological description, originally formulated by Kanizsa in the case of drawings, can be easily adapted to digital images using level lines [1]. The main difference lies in the fact that on drawings, T-junctions occur where the line of the pen meets a previous line only, that is, at places where an object begins to come in front of another. On digital images of natural world, the objects are never uniformly shined, and therefore even 

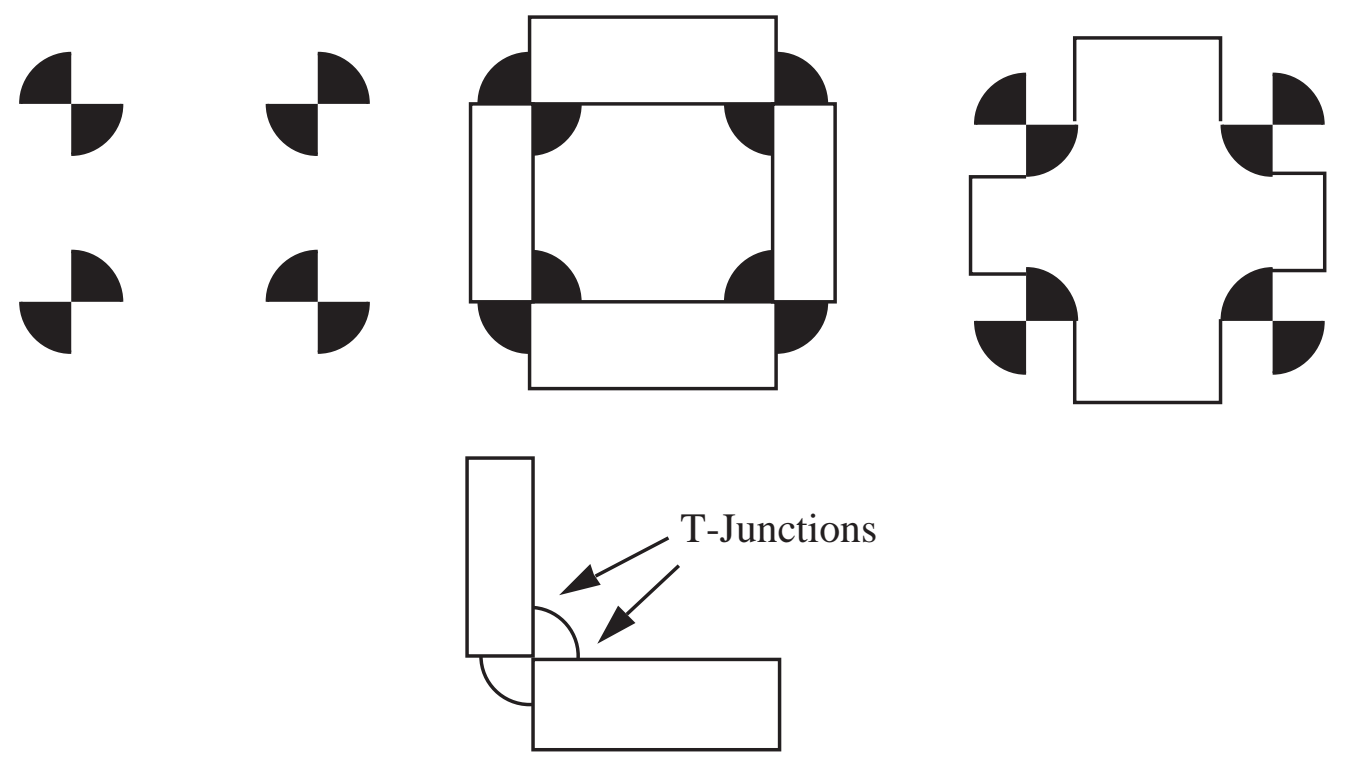

Figure 4. The visual power of T-junctions. Although the first drawing suggests another reconstruction, the human visual system reconstructs the black objects of the second drawing as disks partially covered by boxes. This reconstruction is due to the T-junctions, which are made evident by the topographic map (last drawing). One of the Kanizsa's principle says that the border of the occluded object has to be extended so that to preserve its curvature.

unvaried colored surfaces present lot of level lines. At the borders of an object, these level lines meet the level lines of the background and generate multiple T-junctions: occlusions occur along all the borders (see Fig. 5). The shapes of the objects is then essentially characterised by the T-junctions on them, and by the pieces of the level lines joining these junctions. In this way, one gives a morphological definition of edges:

Definition 5. We call morphological edge a piece of level line joining any number of T-junctions.

The more a morphological edge contains T-junctions, the more it is perceptually significant.

On natural images, level lines are often very close to each other, and the quantization noise leads the curves to join continually: it is therefore essential to introduce some conditions to distinguish between true junctions and junctions due to noise. These conditions are related to the grey level quantization and to the areas of the three sets around the junctions: the one belonging to the occulting object, the one belonging to the occulted object and the one part of the background. To detect the significant T-junctions, we use the following algorithm adapted from $[7,9]$ :

T-junction detection algorithm (Caselles et al.) Parameters :

- A is the area threshold for the level sets,

- $G$ is the grey level threshold for the input image u.

Step 1: Compute the set $\mathcal{J} \subset \Omega$ of pixels where two level lines meet (in a disk of radius $\sqrt{2}$ );

Step 2: for each $x \in \mathcal{J}$, consider the neighbourhood $\mathcal{V}(x)$ of four pixels where the level lines junction is, and 


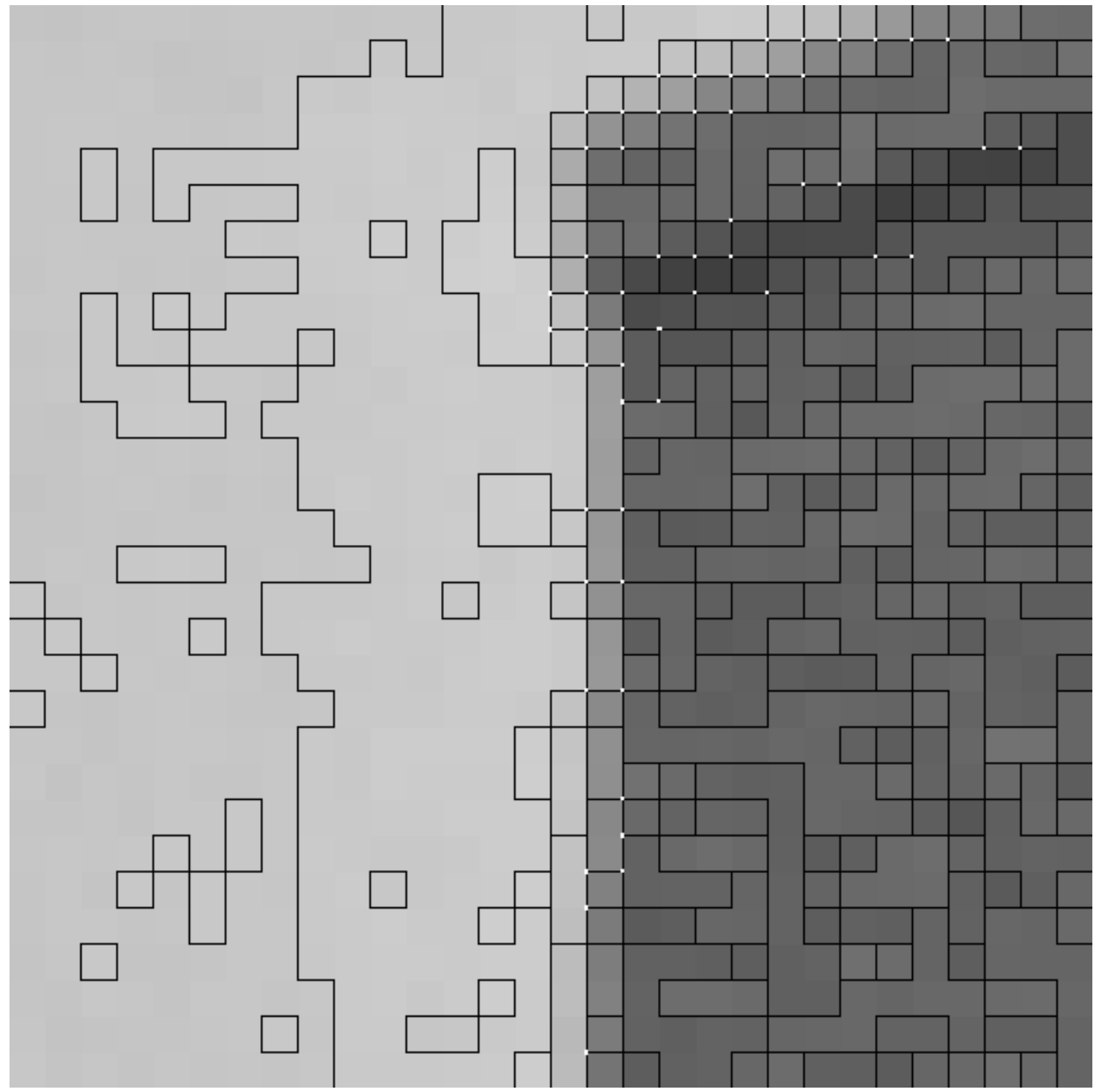

FiguRE 5. Zoom on the upper-left part of the house's chimney, and associated topographic map. The significant T-junctions (computed by the T-junction detection algorithm) are marked by white dots. Notice that they are located at the borders of the important structures.

compute

$-x_{\lambda_{0}}=\arg \inf \{u(y) / y \in \mathcal{V}(x)\}$,

- $\lambda_{0}=u\left(x_{\lambda_{0}}\right)$,

- $x_{\mu_{0}}=\arg \sup \{u(y) / y \in \mathcal{V}(x)\}$,

- $\mu_{0}=u\left(x_{\mu_{0}}\right)$,

- $L_{\lambda}^{k}=$ connected component of $\{y \in \Omega / u(y) \leq \lambda\}$ such that $x_{\lambda_{0}} \in L_{\lambda}^{k}$,

- $M_{\mu}^{k}=$ connected component of $\{y \in \Omega / u(y) \geq \mu\}$ such that $x_{\mu_{0}} \in M_{\mu}^{k}$,

$-\lambda_{1}=\arg \inf \left\{\lambda \geq \lambda_{0} /\right.$ area $\left.\left(L_{\lambda}^{k}\right) \geq A\right\}$,

- $\mu_{1}=\arg \sup \left\{\lambda_{0}<\mu \leq \mu_{0} / \operatorname{area}\left(M_{\mu}^{k}\right) \geq A\right\}$. 
The pixel $x$ is said to be a T-junction if the previous sets and values exist and if the following conditions are meet:

$$
\begin{aligned}
& \text { - } \mu_{1}-\lambda_{1} \geq 2 G, \\
& \text { - } \quad \text { there exists a connected component } K \text { of }\left\{y \in \Omega / \lambda_{1}+G \leq u(y) \leq \mu_{1}-G\right\} \\
& \quad \text { containing a pixel of } \mathcal{V}(x) \text { with area greater than } A .
\end{aligned}
$$

This algorithm ensures that, at every T-junction $x$, there exist significant connected components $L_{\lambda_{1}}^{k}, M_{\mu_{1}}^{k}$ and $K$ with all of them a pixel belonging to $\mathcal{V}(x)$ : the three components are meeting at the junction, and in a neighbourhood of $x$, each component has a common border with another one (see Fig. 6). Therefore, the geometry of each T-junction is characterized by the border of any pair of these three components. To build our compact image model, the connected components $L_{\lambda_{1}}^{k}$ and $M_{\mu_{1}}^{k}$ will be considered. In this way, morphological edges will be composed by borders of these level sets. Notice that at every T-junction $x$ are associated the pixels $x_{\lambda_{0}} \in \partial^{I} L_{\lambda_{1}}^{k}$ and $x_{\mu_{0}} \in \partial^{I} M_{\mu_{1}}^{k}$.

\section{The morphological COMPACt IMAGE MODEL}

The compact image model is based on the discrete morphological decomposition given in Section 2.3, together with a structure of data adapted for compression. Our model contains two types of data: geometrical data record pixel locations in the grid, and numerical data are related to the gray level values at these locations.

\subsection{Geometrical data}

The geometrical data have to code a morphological segmentation $\mathcal{P}$, and we want to compute the $P_{i}$ so that $\partial P_{i}$ are composed by morphological edges. Since each morphological edge belongs to a border of a set $L_{\lambda_{1}}^{k}$ or $M_{\mu_{1}}^{k}$, the issue is to choice, from all possible connected components $K$ of the level sets $L_{\lambda}=\{x \in \Omega / u(x) \leq \lambda\}$ and $M_{\mu}=\{x \in \Omega / u(x) \geq \mu\}$, the ones that contain the greatest numbers of T-junctions or, more generally, the ones that minimize a cost function $\mathcal{C}(K)$, the value $\mathcal{C}(K)$ being small when the border of $K$ matches the border of an object. This cost function may be user-defined; for example, $\mathcal{C}(K)$ may include the cost to code the information related to $K$ in the model.

The following algorithm explains how to solve the first step of the morphological decomposition:

\section{Morphological segmentation algorithm}

Parameters:

$$
\text { - } C \text { is an optional threshold for admissible cost, take } C=+\infty \text { for no parameter. }
$$

Step 1: Decompose the image u into its level sets $\left(L_{\lambda}\right)_{\lambda}$ and $\left(M_{\mu}\right)_{\mu} ;$ initialise $\mathcal{S}$ to contain $\Omega$;

Step 2: using the T-junction detection algorithm, compute the sequences of T-junctions $T_{0}=\left(x_{\lambda_{0}}\right)_{\lambda_{0}}$ and $T_{1}=\left(x_{\mu_{0}}\right)_{\mu_{0}}$;

Step 3: put in $\mathcal{S}$ the connected component with minimal cost of type $L_{\lambda}^{k}$, as follows.

$$
\begin{aligned}
& -\quad \text { Compute (for example) } \forall \lambda, k, \mathcal{C}\left(L_{\lambda}^{k}\right)=1 /\left(\left|T_{0} \cap \partial^{I} L_{\lambda}^{k}\right|\right), \\
& -\quad \bar{\lambda}, \bar{k}=\arg \inf \mathcal{C}\left(L_{\lambda}^{k}\right), \\
& -\quad \text { if } \mathcal{C}\left(L_{\frac{\bar{k}}{\lambda}}^{\bar{k}}\right)<C, \text { then set } \mathcal{S} \leftarrow \mathcal{S} \cup L_{\frac{\bar{k}}{\lambda}}^{\bar{k}}, T_{0} \leftarrow T_{0} \backslash \partial^{I} L_{\bar{\lambda}}^{\bar{k}}
\end{aligned}
$$

Step 4: Put in $\mathcal{S}$ the connected component with minimal cost of type $M_{\mu}^{k}$, as follows.

$$
\begin{aligned}
& \text { - Compute (for example) } \forall \mu, k, \mathcal{C}\left(M_{\mu}^{k}\right)=1 /\left(\left|T_{1} \cap \partial^{I} M_{\mu}^{k}\right|\right), \\
& -\bar{\mu}, \bar{k}=\arg \inf \mathcal{C}\left(M_{\mu}^{k}\right), \\
& -\quad \text { if } \mathcal{C}\left(M_{\bar{\mu}}^{\bar{k}}\right)<C, \text { then set } \mathcal{S} \leftarrow \mathcal{S} \cup M_{\bar{\mu}}^{\bar{k}}, T_{1} \leftarrow T_{1} \backslash \partial^{I} M_{\bar{\mu}}^{\bar{k}} .
\end{aligned}
$$




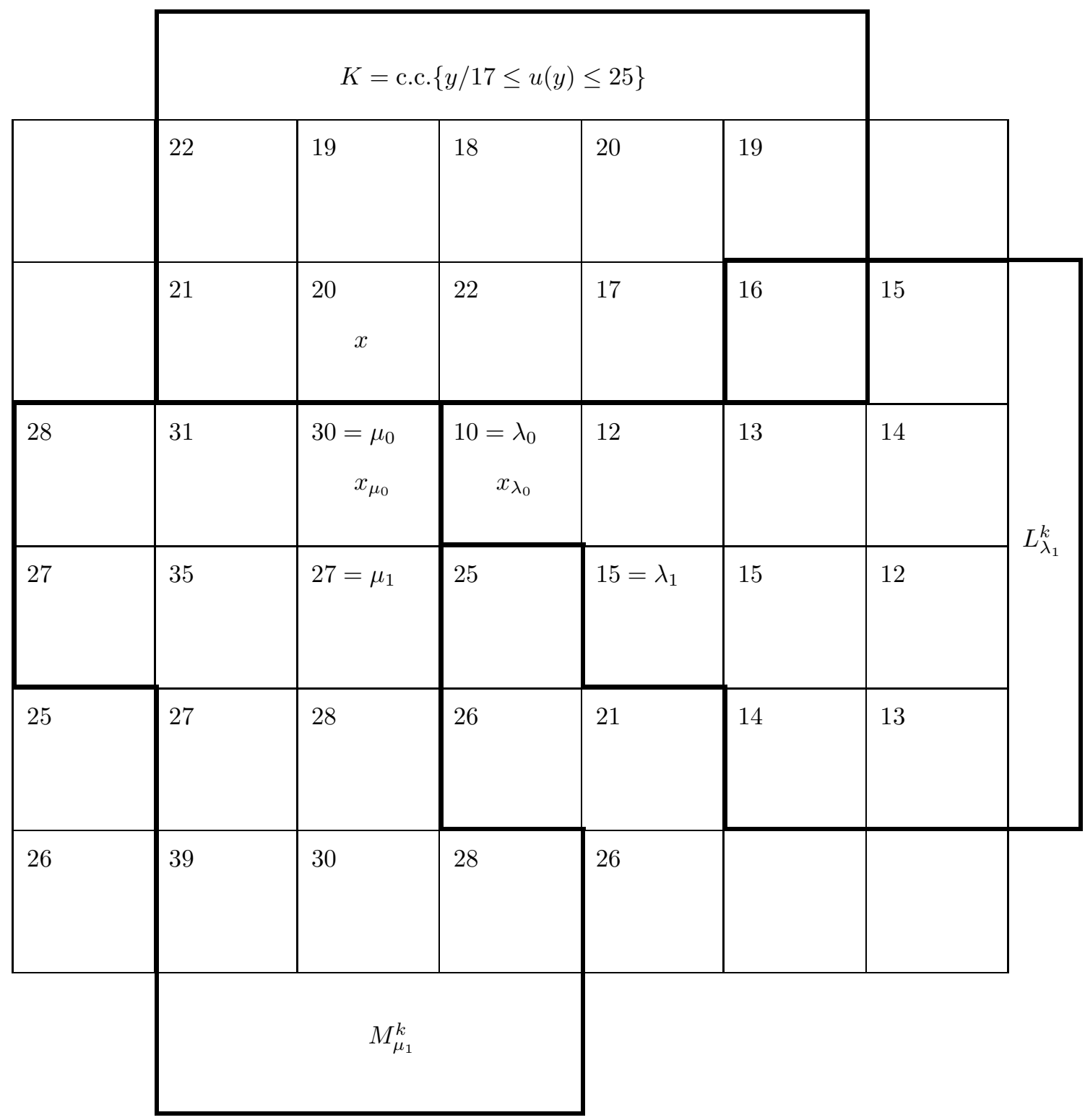

Figure 6 . The T-junction detection algorithm ensures significant connected components $L_{\lambda_{1}}^{k}$, $M_{\mu_{1}}^{k}$ and $K$ associated to each retained T-junction $x$. The number written in each box indicates the gray level of the pixel. In this example, the parameters are $A=8$ and $G=2$.

Step 5: Loop to step 3 while $\inf \left\{\mathcal{C}\left(L_{\bar{\lambda}}^{\bar{k}}\right), \mathcal{C}\left(M_{\frac{\bar{k}}{\mu}}\right)\right\}<C$.

When this algorithm ends, $\mathcal{P}=\operatorname{MS}(\mathcal{S})$ is a morphological segmentation of $u$ so that each $\partial P_{i}$ is made by pieces of morphological edges. An important fact to notice is that the most perceptive edges are recorded first. The resolution of the segmentation, that is, the visual significance of the less perceptive edge, is given by the 
parameters $A$ and $G$ of the T-junction algorithm and, eventually, by the maximal cost $C$. Figure 2 gives the result of this algorithm on the "House" image, with $C=+\infty$.

\subsection{Numerical data}

The numerical data are used to compute the smooth and non-oscillating approximation $v$ on $u$ defined on each $P_{i}$. The morphological segmentation algorithm ensures that no important edge can be located inside $P_{i}$ : this explains why the image may be well approximated by a piecewise-smooth function $v$. In addition, the approximating function is chosen non-oscillating so that it catches the sketch and not the textures, according to the morphological decomposition. How should be chosen the data to allow a good approximation? Since the border of $P_{i}$ is made by morphological edges, the knowledge of $u$ in the internal and external side of each edge is the basic information. In order to get a compact model, we propose to retain only two samples of $u$ for each morphological edge (one for the internal side and one for the external). The value are chosen in order to lower the variations of $v$ between two neighbouring regions of a T-junction. This not only helps to get a non-oscillating function, but also prevents the appearance of visual artefacts near the edges by keeping a low contrast.

A pixel $x$ of $\partial^{I} P_{i}$ may be associated to several morphological edges (parts of different lower level sets or different upper level sets). In that case, $v(x)$ is set to be the closest value to $u(x)$. This operation corresponds to chose the smallest level set.

To compute the values $\phi_{i}(x)$ of $v$ on each $\partial^{I} P_{i}$, we use the following algorithm:

Algorithm to get samples of the approximating function

Step 1: Compute $v$ on internal border associated to lower level sets, as follows.

$\forall P_{i} \in \mathcal{P}=M S(\mathcal{S}), \forall x \in \partial^{I} P_{i}$, if $x$ belongs to an internal border of a connected component

of a lower level set of $\mathcal{S}$, and if $\phi_{i}(x)$ has not been already defined, define

$$
\phi_{i}(x)=\inf _{\lambda, l / x \in \partial^{I} L_{\lambda}^{l}} \sup _{y \in \partial^{I} L_{\lambda}^{l}} u(y) \text {. }
$$

Step 2: Compute $v$ on internal borders associated to upper level sets, as follows.

$$
\begin{aligned}
& \forall P_{i} \in \mathcal{P}=M S(\mathcal{S}), \forall x \in \partial^{I} P_{i} \text {, if } x \text { belongs to an internal border of a connected component } \\
& \text { of an upper level set of } \mathcal{S} \text {, and if } \phi_{i}(x) \text { has not been already defined, define } \\
& \qquad \phi_{i}(x)=\sup _{\mu, l / x \in \partial^{I} M_{\mu}^{l}} \inf _{y \in \partial^{I} M_{\mu}^{l}} u(y) \text {. }
\end{aligned}
$$

When this algorithm ends, a value $\phi_{i}(x)$ has been defined at each point $x$ of each $\partial^{I} P_{i}$ that is, according to the proposition 3, at each left and right hand sides of any curve of $\partial P_{i}: \phi_{i}$ is therefore a function defined on $\partial^{I} P_{i}$.

To summarize our discussion, the compact image model contains the following data:

- the morphological segmentation map $\left(\partial P_{i}\right)_{i}$;

- the sequence of samples $\left(\phi_{i}(x)\right)_{i ; x \in \partial^{I} P_{i}}$.

\section{Reconstruction of images From the MODEL}

The issue of computing an approximating function $v$ from the samples $\left(\phi_{i}(x)\right)_{i ; x \in \partial^{I} P_{i}}$ belongs to the class of interpolation problems. Different approaches using image interpolation techniques have been described in the literature (see for example $[5,16,21]$ ), some of them including the same motives than ours of catching the image sketch in a compact way. Recently, a morphological interpolation technique for image coding has been proposed by Casas in [6]. In our knowledge, our work is the first one to use a segmentation map not based on a classical (and non morphological) edge detector, but on a selection of the level sets that carry the atoms of the perception.

We shall retain the work of Caselles, Morel and Sbert in [8], where they extend the Casas' morphological interpolation technique, and where they prove that any interpolation operator (satisfying fairly conditions, such 
as morphological, invariance and regularity properties) comes down to let evolve the interpolating function $r_{i}$ on each $P_{i}$ with the following equation:

$$
\begin{cases}\frac{\partial w}{\partial t}=D^{2} w\left(\frac{D w}{|D w|}, \frac{D w}{|D w|}\right) & \forall t>0, \forall x \in P_{i} ; \\ w(0, x)=w_{0}(x) & \forall x \in P_{i} ; \\ w(t, x)=w_{0}(x)=\phi_{i}(x) & \forall t>0, \forall x \in \partial P_{i} .\end{cases}
$$

We have written by $D w$ the gradient of $w$ along the spatial coordinates, and by $D^{2} w$ the Hessian of $w$, that is, the matrix of the second derivatives of $w$.

Under some reasonable conditions [3], there exists a unique continuous viscosity solution $w(t, x)$ of $(24)$ such that $w(t,$.$) is a Lipshitz function for all t>0$ on each $P_{i}$, with uniformly bounded Lipschitz norm. When $t \rightarrow+\infty, w(t,.) \rightarrow r_{i}$ with $r_{i \mid \partial P_{i}}=\phi_{i}$. The function $r_{i}$ is an absolutely minimizing Lipschitz interpolant of $\phi_{i}$ inside $P_{i}$, or AMLE for short.

As in the Proposition 2, the function $v$ is defined by $v=r_{i}$ on each region $P_{i}$ of the morphological segmentation $\mathcal{P}$. It is a non-oscillating piecewise-smooth function that verifies $\left.v\right|_{\partial P_{i}}=\phi_{i}$ (or $\left.v\right|_{\partial^{I} P_{i}}=\phi_{i}$ in the discrete case) and which is, in the sense given by the sampling algorithm in Section 4.2, an approximation of $u$.

The evolution equation (24) can be solved using an implicit Euler scheme, so that to transform the evolution problem to a sequence of non linear elliptic problems, which leads in a discrete case to an implicit difference scheme.

\section{TeXture REFinement AND COMPRESSiON}

\subsection{Texture refinement}

The pertinence of a morphological decomposition is connected to the quality of the sketch approximation $v$ of the original image $u$. Because of the imperfect approximation (mainly due to the limited information furnished by the samples $\left(\phi_{i}(x)\right)_{i ; x \in \partial^{I} P_{i}}$, , some low-frequency information is not catched in $v$ and appears in the error image $w=u-v$, whereas it does not participate in the texture information. As noticed in Section 2.1, some level sets with irregular borders may also be included in $w$ and they should be removed.

The texture refinement consists in the detection of this unwanted information, and it is based on the following observations:

- the microtexture information is associated to fast oscillations of the image which create level sets of small sizes, whereas low-frequency information due to the imperfect approximation creates level sets of bigger sizes;

- level lines can be made smooth by slightly pre-filtering the image $u$ using a morphological filter, such as MCM (mean curvature motion) or AMSS [2]. Let us denote by $F(u)$ this pre-filtered image, which differs from $u$ at level set borders only.

\section{Texture refinement algorithm}

Step 1: Compute the discrete morphological decomposition on the filtered image $F(u)$. Let $w=u-v$ be the difference image between the original and the sketch $v$ obtained from $F(u)$.

Step 2: Remove from $w$ all level sets of big sizes (low-frequency information) and set gray level values to 0 in the neighbourhood of the level lines $\left(\partial P_{i}\right)_{i}$. Let $w_{R}$ be the resulting image, called refined microtexture image.

Step 3: Compute the impurity image $w_{S}=w-w_{R}$ which carries mainly the remaining sketch information of $u$.

\subsection{Texture compression}

The compact image model allows to compress the original image at very low bit rates, by coding the segmentation map $\left(\partial P_{i}\right)_{i}$ and the samples $\left(\phi_{i}(x)\right)_{i ; x \in \partial^{I} P_{i}}$ that define the sketch image $v$. However, since 
this compression scheme removes all the microtextures, the reconstructed image $v$ may not sound natural. To recover some of the texture information, we can compress the error image $w$ or, for better results, the refined microtexture image $w_{R}$. While the information contained in the sketch image is essentially of geometrical structure, microtextures are better characterised in the space-frequency domain. It is well known that, among linear scale-space based compression schemes, biorthonormal wavelet decompositions [12] offer one of the best results. We use a variant of the standard Shapiro's EZW (Embedded Zerotrees Wavelet) algorithm [34] to compress the microtexture image with biorthonormal wavelet bases. This choice may not be optimal and improved results may be obtained with bases better localized in the frequency domain, as wavelet packets or local trigonometric bases $[22,27]$.

To compress natural images containing edges, schemes based on a wavelet representation offer better results than the ones based on Fourier, but Gibbs phenomena errors due to the quantization and the sampling of the coefficients might still appear at high compression ratios. These errors are located where the wavelet coefficients decrease slowly, that is, at the discontinuity lines. Therefore, the most perceptive errors are located at the neighbourhood of the main level set borders, which are given by $\left(\partial P_{i}\right)_{i}$. Thanks to the texture refinement algorithm, the refined microtexture image $w_{R}$ does not carry any information at these locations. For these reasons, we expect better quality in the compressed refined image $\widehat{w_{R}}$ than in the compressed image $\widehat{w}$.

\section{ExPERIMENTAL RESUlts}

All algorithms given in this paper have been implemented in the MegaWave2 image processing environment [17]. In order to show how the compact image model can generate a high compression scheme, data have been error-free compressed using adapted coding techniques.

Figure 7 summarises the experimental results obtained with the "House" image $u$, from the morphological segmentation presented in Figure 2. With this choice of morphological segmentation that retains the very most important structures only, the data of the image model are extremely compact: a compression ratio of 38 is achieved. However, since all important objects are well preserved, one can easily recognized the original image on the sketch image $v$. The vanishing information is given by the image difference $w=u-v$. As expected, the image $w$ carries the microtextures of the original image $u$ : the textures of the brick wall and of the sky are particularly well catched. As noticed in Section 6.1, one can see some low-frequency information in addition to the microtextures (especially along the right side border of the roof). These patterns are due to the samples $\left(\phi_{i}(x)\right)_{i ; x \in \partial^{I} P_{i}}$ used to reconstruct the image values in each region $P_{i}$ : the average of $u$ into $P_{i}$ does not necessary equal the average of $v$. However, this does not affect the visual quality of the reconstructed image $v$. Indeed, the gray level of a region is not relevant by itself, but by comparisons with the level of the neighbouring regions only. The algorithm that computes the samples $\left(\phi_{i}(x)\right)_{i ; x \in \partial^{I} P_{i}}$ (see Sect. 4.2) has been composed so that to satisfy this local comparison principle.

Depending on the complexity of the morphological segmentation, the sketch image may contain much more information that the one presented the Figure 7. It is even possible to keep some structures that may be interpreted as microtextures, leading to a sketch image visually identical to the original. This progressive representation property is illustrated by Figure 8, where the complexity of the sketch image is increased with the number of morphological edges.

We shall now present some results related to texture refinement and compression. The up-left image of Figure 9 has to be compared to the down-right image of Figure 7. The first one is the refined microtexture image $w_{R}$ while the second one is the raw microtexture image $w$. The difference between them is given by the image $w_{S}$, displayed in the up-right part of Figure 9. As expected, the refined microtexture image does not contain the low-frequency structures that we have noticed in $w$, and which are well isolated in $w_{S}$. Regarding the parameters choosen to compute the morphological decomposition, the remaining sketch image $w_{S}$ does not contain relevant information. We propose to ignore this image in the texture compression scheme.

Figure 10 presents the results obtained by compressing the refined microtexture image $w_{R}$, while Figure 11 gives the results with the raw microtexture image $w$. Both compressions are made at the same bit rate. We 

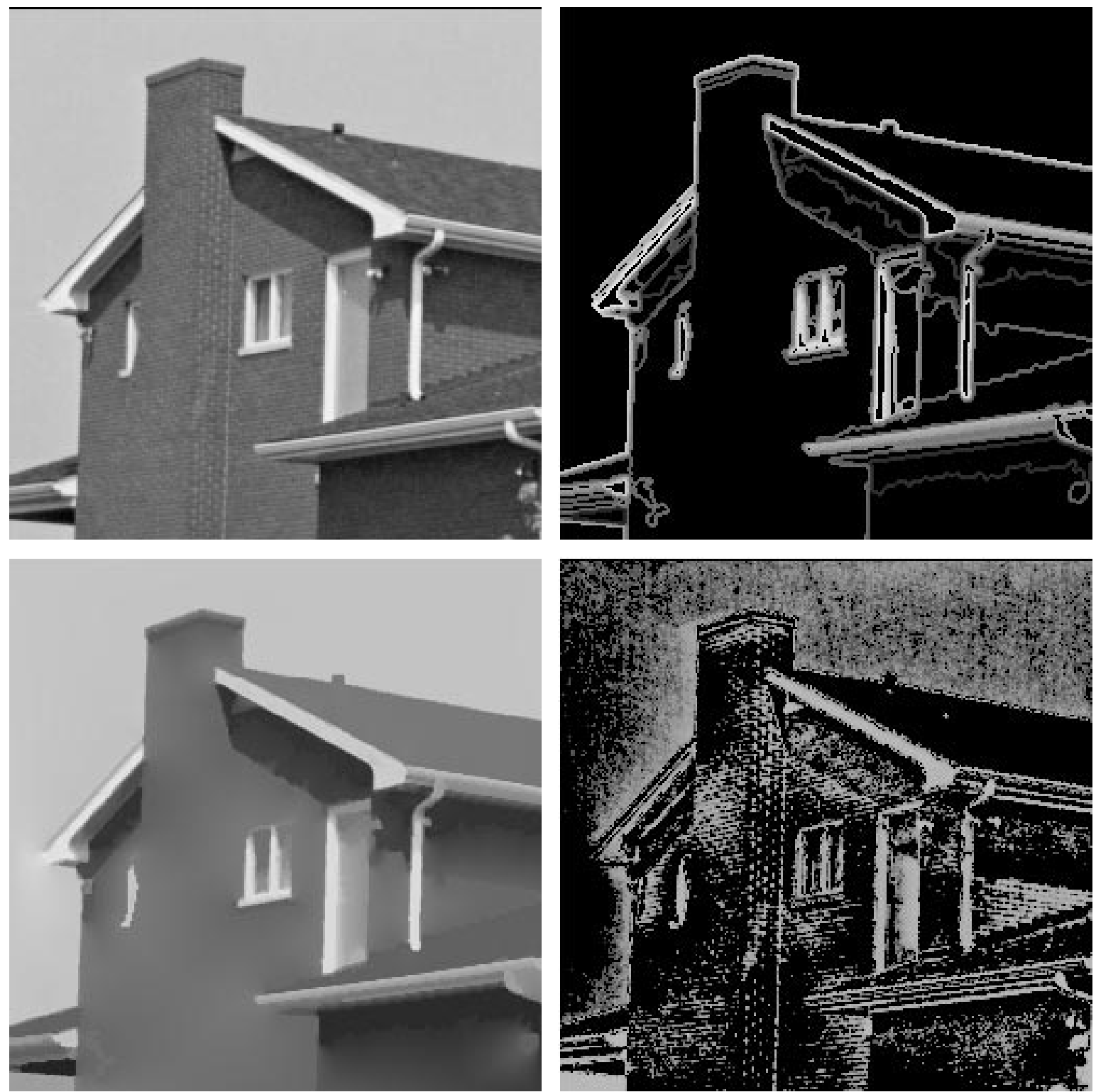

Figure 7. Compact image model of the "House" image. Up and left: original "House" image $u$. $\mathrm{TV}(u) \simeq 8 \times 10^{5},\|u\|_{1} \simeq 9 \times 10^{6}$. Up and right: compact sketch model with 194 morphological edges. In this image, the samples $\left(\phi_{i}(x)\right)_{i ; x \in \partial^{I} P_{i}}$ are bitmapped at the pixels borders $\partial^{I} P_{i}$ of the segmentation map. By coding this image, one can achieve a total bit rate of $0.21 \mathrm{bpp}$ (bit per pixel), which corresponds to a compression ratio of 38. Down and left: reconstruction from the previous image, using the AMLE model. This is the sketch image $v$. TV $(v) \simeq 2 \times 10^{5}$, $\|v\|_{1} \simeq 9 \times 10^{6}$. Down and right: error image $w=u-v$. TV $(w) \simeq 7 \times 10^{5},\|w\|_{1} \simeq 6 \times 10^{5}$. This image contains the microtexture and some remaining sketch information. To print this signed image, the pixels absolute value has been considered and a change of contrast has been applied. 

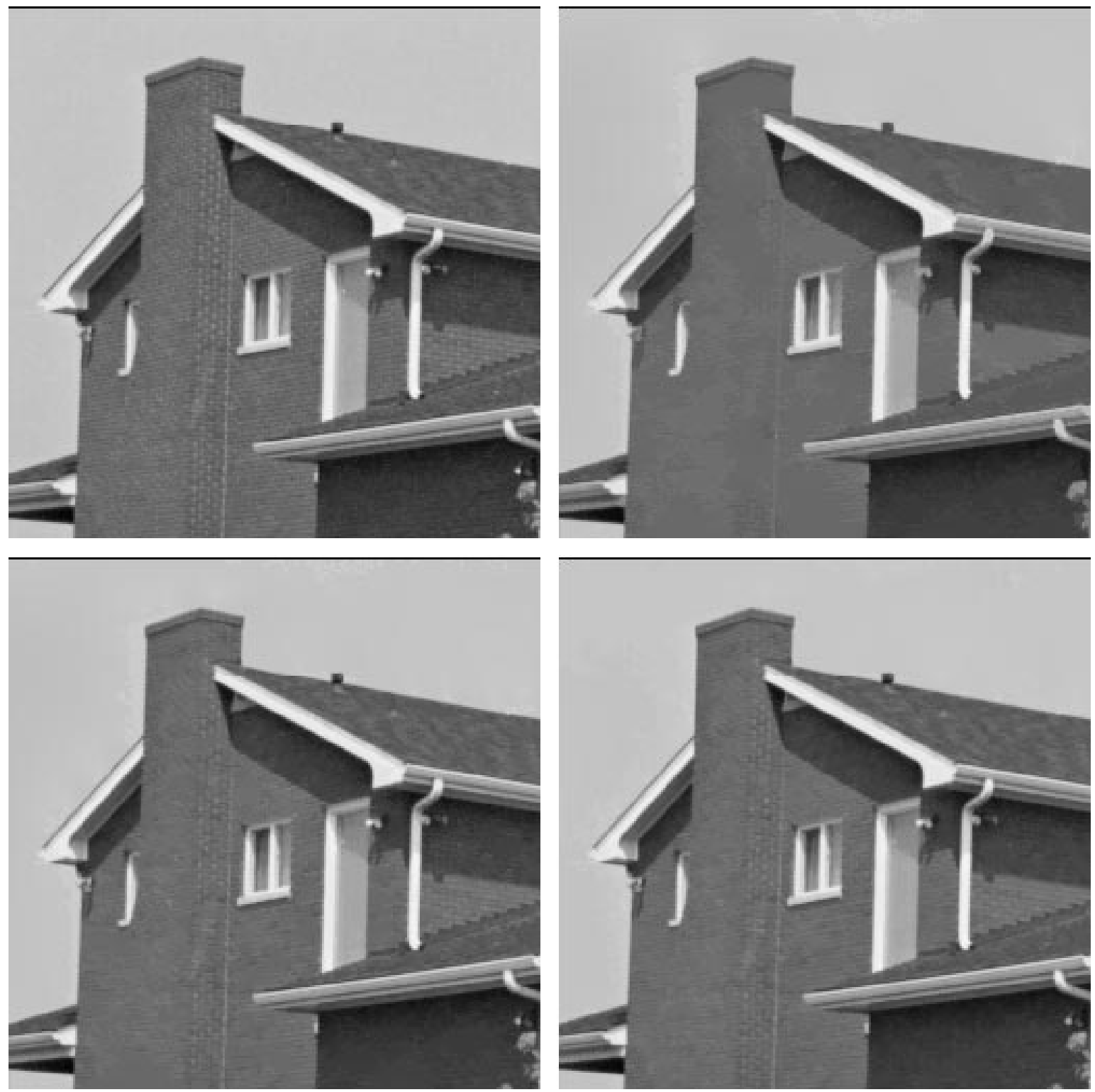

FiguRE 8. Different sketch images obtained by increasing the number of morphological edges considered as perceptually significant. Up and left: original "House" image $u$; TV $(u) \simeq 8 \times 10^{5}$. Up and right: sketch image with 1928 morphological edges; $\operatorname{TV}(v) \simeq 5.4 \times 10^{5}$. Down and left: sketch image with 3760 morphological edges; $\mathrm{TV}(v) \simeq 6.5 \times 10^{5}$. Down and right: sketch image with 10105 morphological edges; $\mathrm{TV}(v) \simeq 6.6 \times 10^{5}$.

notice a better visual quality in the compressed textured sketch image $v+\widehat{w_{R}}$ than in the compressed original image $\widehat{u}=v+\widehat{w}$ : the microtextures are better compressed and there is no noticeable Gibbs artifacts. 

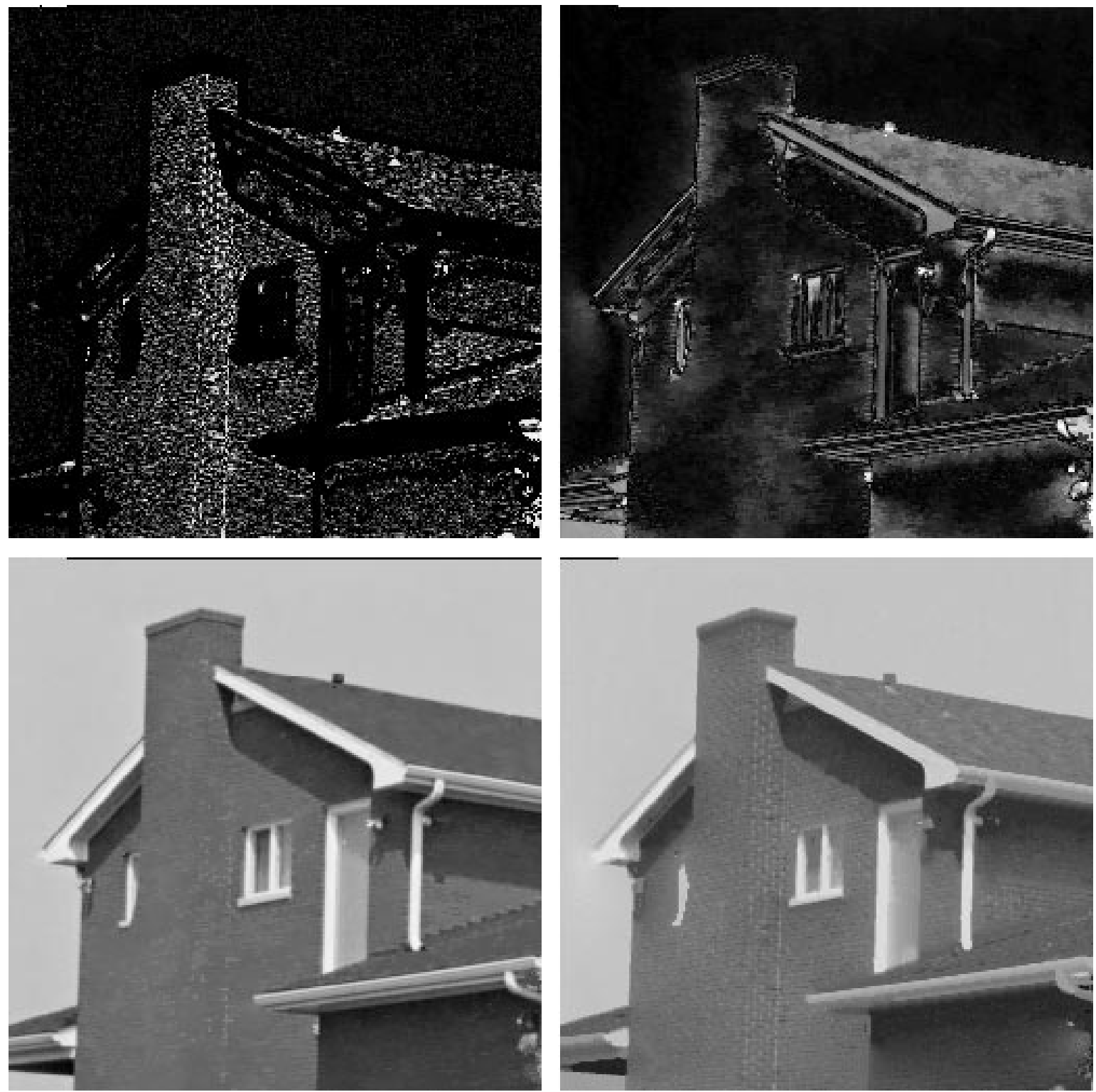

Figure 9. Texture refinement and full sketch extraction. Up and left: refined microtexture image $w_{R}$ obtained by removing the remaining sketch information in $w$. Up and right: remaining sketch image $w_{S}=w-w_{R}$. This image carries the average gray levels differences between the original image $u$ and the sketch image $v$, together with the shapes that have not been retained because of their negligible morphological edges. We claim that this information is perceptually irrelevant (regarding the parameters choosen) and may be thrown away. Down and left: full sketch image $v+w_{S}$. Down and right: textured sketch image $v+w_{R}=u-w_{S}$. This is the original image without the remaining sketch image $w_{S}$. To print this signed image (up left and right), the pixels absolute value has been considered and a change of contrast has been applied. 

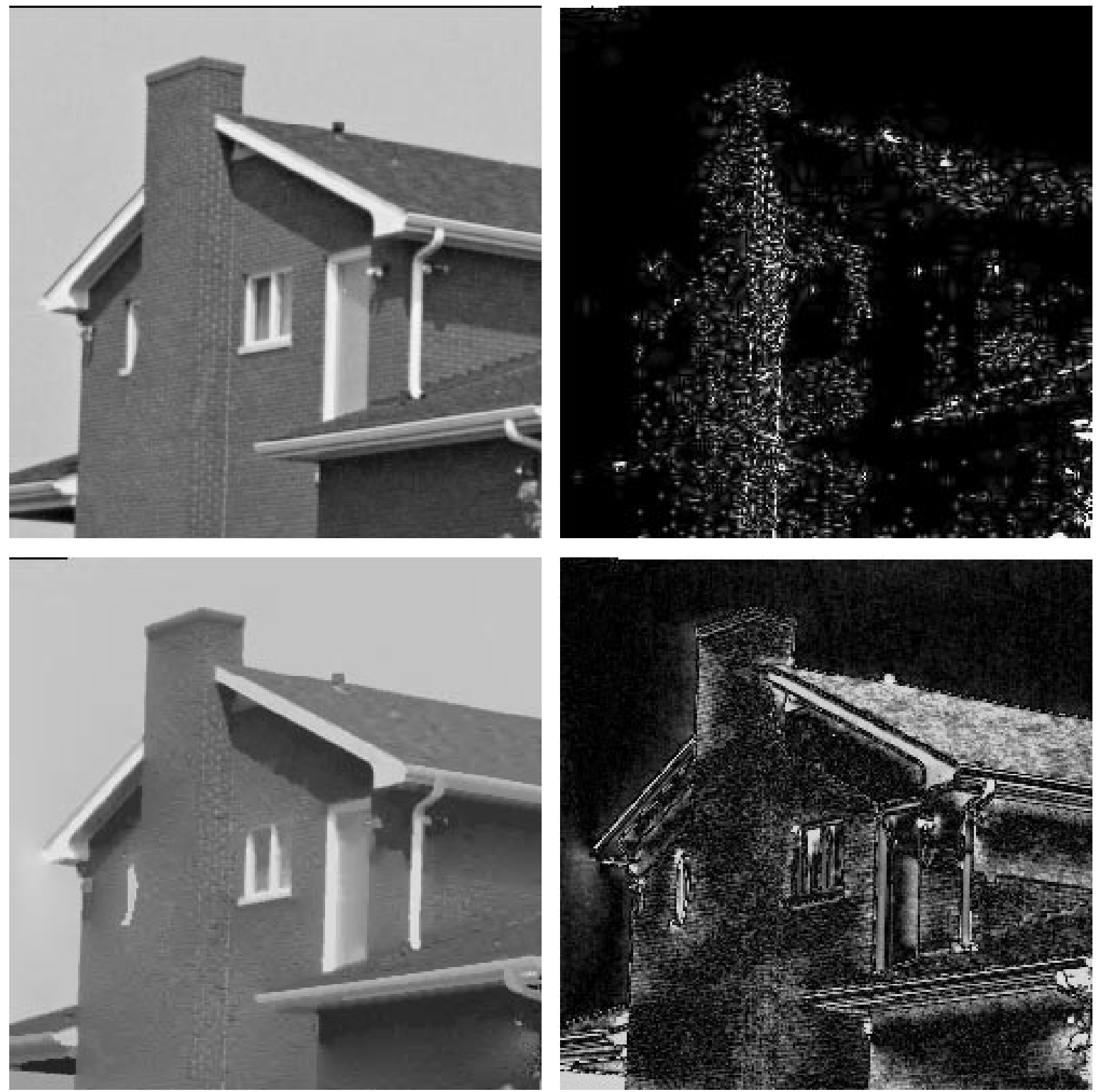

Figure 10. Compression experiment on the textured sketch image (without $w_{S}$ ). Up and left: original "House" image $u$. Up and right: compressed refined microtexture image $\widehat{w_{R}}$, using EZW algorithm. Bit rate $=0.29$ bpp. Down and left: compressed textured sketch image $v+\widehat{w_{R}}$. Total bit rate $=0.5$ bpp (compression ratio: 16). Down and right: error $u-\left(v+\widehat{w_{R}}\right)$. To print this signed image (right up and down), the pixels absolute value has been considered and a change of contrast has been applied.

\section{Conclusion}

Most of compact image models that can be found in the literature, and which tend to split the information between sketch and texture, are based on the detection of the most important discontinuity lines in the image $[5,16,21]$ (recently, an alternate way to efficiently compress the various structures of natural images has been 

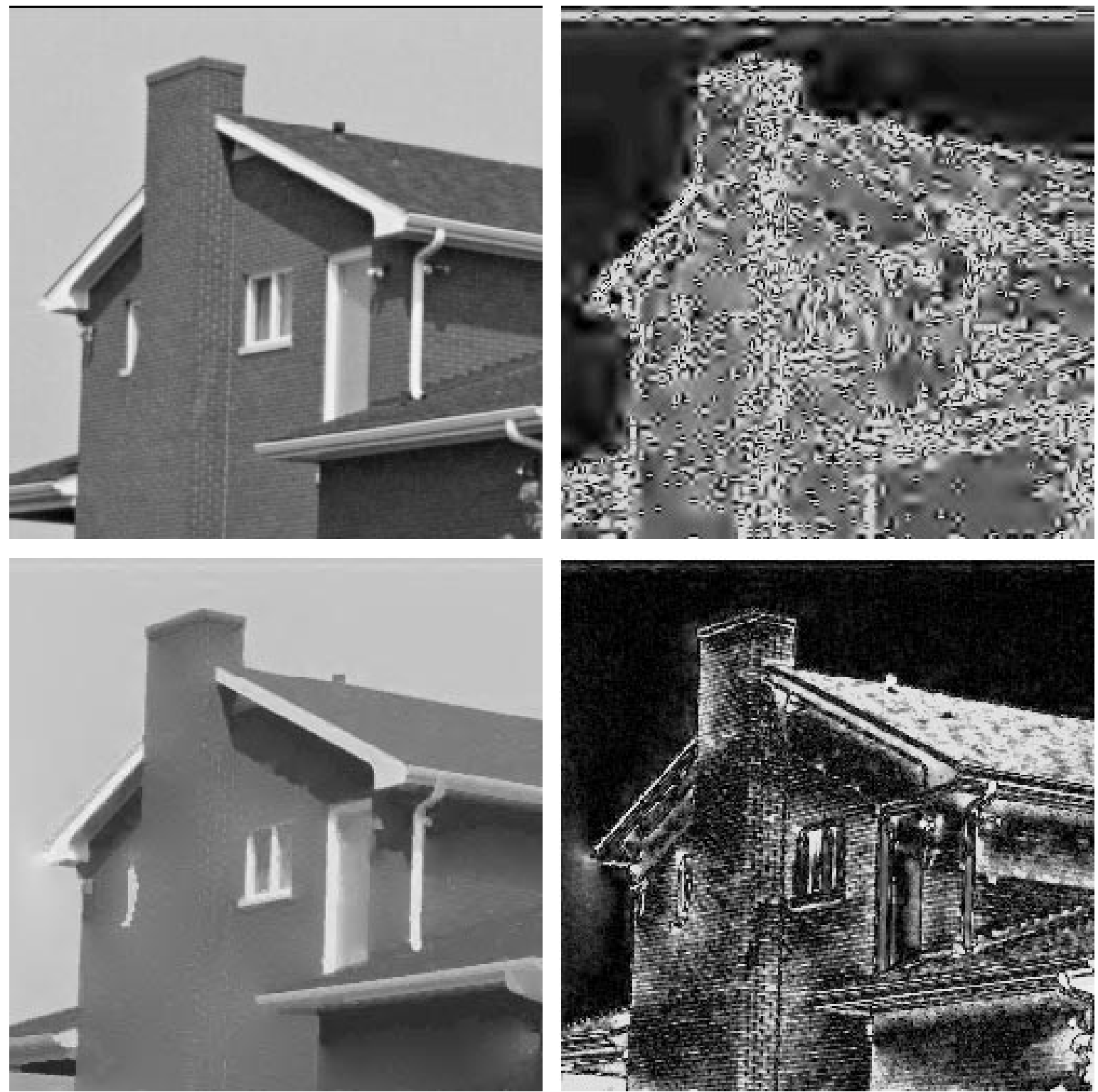

FIGURE 11. Compression experiment on the original image (with $w_{S}$ ). Up and left: original "House" image $u$. Up and right: compressed error image $\widehat{w}$, using EZW algorithm. Bit rate $=0.29 \mathrm{bpp}$. Notice the important Gibbs phenomena due to number of discontinuity lines. Down and left: compressed original image $\widehat{u}=v+\widehat{w}$. Total bit rate $=0.5 \mathrm{bpp}$ (compression ratio: 16). On a high resolution screen, the Gibbs phenomena from $\widehat{w}$ are still visible in $\widehat{u}$. Down and right: error image $u-(v+\widehat{w})$. To print this signed image (right up and down), the pixels absolute value has been considered and a change of contrast has been applied.

proposed in [27]. This approach, which consists of using different space-frequency bases to encode the different structures, does not require the detection of edges). In our paper, we propose a new definition of edges, as level lines containing a great number of topological singularities (the T-junctions). Using the total variation, 
we explain why the microtexture can be removed by approximating the image with a piecewise smooth nonoscillating function, the singularities being located at the morphological edges. This approach allows to build an image model respectful of the human visual system, and with a compactness that makes it suitable to perform image compression at low bit rate.

The author would like to thank Jean-Michel Morel, Françoise Dibos and Jean-Pierre D'Alès for valuable discussions. The AMLE interpolation algorithm has been implemented with the help of Catalina Sbert and Jean-Pierre D'Alès. The T-junction detection algorithm has been implemented with the help of Vicent Caselles, Bartomeu Coll and José Luis Lisani. The EZW compression scheme has been implemented by Jean-Pierre D'Alès.

\section{REFERENCES}

[1] J.P. D'Alès, J. Froment and J.M. Morel, Reconstruction visuelle et généricité, Second European Workshop on Image Processing and Mean Curvature Motion, UIB, Spain (1995) 1-21.

[2] L. Alvarez, F. Guichard, P.L. Lions and J.M. Morel, Axioms and fundamental equations of image processing. Arch. Rational Mechanics and Anal. 16 (1993) 200-257.

[3] F. Cao, Absolutely minimizing Lipschitz extension with discontinuous boundary data. C.R. Acad. Sci. Paris 327, Série I (1998) 563-568.

[4] J. Canny, A computational approach to edge detection. IEEE Trans. Pattern Analysis and Machine Intelligence 8 (1986) 679-698.

[5] S. Carlsson, Sketch based coding of grey level images. Signal Processing North-Holland 15 (1998) 57-83.

[6] J.R. Casas, Morphological Interpolation for Image Coding, 12th International Conference on Analysis and Optimization of Systems. Images, Wavelets and PDEs. M.O. Berger, R. Deriche, I. Herlin, J. Jaffré, J.M. Morel, Eds., Springer, Paris (1996).

[7] V. Caselles, B. Coll and J.M. Morel, A Kanizsa programme. Progress in Nonlinear Differential Equs. and their Applications 25 (1996) 35-55.

[8] V. Caselles, J.M. Morel and C. Sbert, An Axiomatic Approach to Image Interpolation. IEEE Trans. on Image Processing 7 (1998) 376-386.

[9] V. Caselles, B. Coll and J.M. Morel, Topographic maps and local contrast changes in natural images. Internat. J. Comput. Vision, to appear.

[10] V. Caselles, B. Coll and J.M. Morel, The Connected Components of Sets of Finite Perimeter in the Plane, Preprint (1998).

[11] A. Chambolle and P.L. Lions, Image Recovery via Total Variation Minimization and Related Problems. Numerische Mathematik 76 (1997) 167-188.

[12] A. Cohen, I. Daubechies and J.C. Feauveau, Biorthogonal bases of compactly supported wavelets. Comm. in Pure E Appl. Math. 45 (1992).

[13] M.G. Crandall, H. Ishii and P.L. Lions, User's Guide to Viscosity Solution of Second Order Partial Differential Equations. Bull. Amer. Math. Soc. 27 (1992) 1-67.

[14] F. Dibos and G. Koepfler, Global Total Variation Minimization, TR 9801, CEREMADE, Université Paris-Dauphine, France (1998).

[15] L.C. Evans and R.F. Gariepy, Measure theory and fine properties of functions, Studies in Advanced Mathematics, CRC Press Inc. (1992).

[16] J. Froment and S. Mallat, Second Generation Compact Image Coding with Wavelets, Wavelets - A Tutorial in Theory and Applications, C.K. Chui, Ed., Academic Press (1992) 655-678.

[17] J. Froment, Vol. 1: MegaWave2 User's Guide, Vol. 2: MegaWave2 System Library, Vol. 3: MegaWave2 User's Modules Library, The Preliminary Guides to the MegaWave2 Software (1998). A version is available at http://www.cmla.ens-cachan.fr/Cmla/Megawave/ into the software package.

[18] R. Hummel and R. Moniot, Reconstruction from zero-crossings in scale-space. IEEE Trans. on Acoustic, Speech and Signal Processing 37 (1989).

[19] G. Kanisza, Grammatica del Vedere, Il Mulino, Bologna (1980).

[20] G. Kanisza, Vedere e pensare, Il Mulino, Bologna (1991).

[21] M. Kunt, M. Bénard and R. Leonardi, Recent Results in High-Compression Image Coding. IEEE Trans. on Circuits and Systems (1987) 1306-1336.

[22] J. Li, P.Y. Cheng and C.C.J. Kuo, An embedded wavelet packed transform technique for texture compression. SPIE 2569 (1995) 602-613.

[23] S. Mallat and S. Zhong, Characterization of signals from multiscale edges. IEEE Trans. Pattern Recognition and Machine Intelligence 14 (1992) 710-732.

[24] D. Marr, Vision, W.H. Freeman and Co. (1982).

[25] S. Masnou and J.M. Morel, Level lines based disocclusion, ICIP'98 IEEE Int. Conf. on Image Processing Chicago (1998).

[26] G. Matheron, Random Sets and Integral Geometry, John Wiley, N.Y. (1975). 
[27] F.G. Meyer, A.Z. Averbuch, J-O. Strömberg and R.R. Coifman, Multi-layered image representation: Application to image compression, ICIP'98 IEEE Int. Conf. on Image Processing, Chicago (1998).

[28] Y. Meyer, Wavelets: Algorithms and applications, SIAM (1993).

[29] P. Monasse and F. Guichard, Fast computation of a contrast-invariant image representation, TR 9815, CMLA, ENS Cachan, France, IEEE Trans. on image processing, submitted.

[30] J.M. Morel, Perception visuelle et traitement d'images (I) et (II), Revue du Palais de la Découverte (novembre 1996 et Février 1997).

[31] J.M. Morel and S. Solimini, Variational methods in image processing, Birkhäuser (1994).

[32] L.I. Rudin and S. Osher, Total Variation based image restoration with free local constraints, in Proc. IEEE Int. Conf. on Image Processing 1 (1994) 31-35.

[33] J. Serra, Image analysis and mathematical morphology, Academic Press (1982).

[34] J. Shapiro, Embedded Image Coding Using Zerotrees of Wavelet Coefficients. IEEE Trans. on Signal Processing 41 (1993) $3445-3462$

[35] M. Wertheimer, Untersuchungen zur Lehre der Gestalt, Psychologische Forschung, IV (1923) 301-350. 